\title{
Performance of a novel wafer scale CMOS active pixel sensor for bio-medical imaging
}

\begin{tabular}{|c|c|}
\hline Journal: & Physics in Medicine and Biology \\
\hline Manuscript ID: & PMB-100279.R1 \\
\hline Manuscript Type: & Paper \\
\hline Date Submitted by the Author: & $n / a$ \\
\hline Complete List of Authors: & $\begin{array}{l}\text { Esposito, Michela; University of Surrey, Centre for vision Speech and Signal } \\
\text { Processing; University of Lincoln, School of Computer Science, } \\
\text { Anaxagoras, Thalis; ISDI Ltd (Image sensor design and innovation), } \\
\text { Konstantinidis, Anastasios; University College London, Department of } \\
\text { Medical Physics and Bioengineering } \\
\text { Zheng, Yi; University College London, Department of Medical Physics and } \\
\text { Bioengineering } \\
\text { Speller, Robert; University College London, Department of Medical Physics } \\
\text { and Bioengineering } \\
\text { Evans, Philip; University of Surrey, Centre for Vision Speech and Signal } \\
\text { Processing Faculty of Engineering and Physical Sciences } \\
\text { Allinson, Nigel; University of Lincoln, School of Engineering } \\
\text { Wells, Kevin; University of Surrey, Centre for vision Speech and Signal } \\
\text { Processing }\end{array}$ \\
\hline Article Keywords: & $\begin{array}{l}\text { CMOS Active Pixel Sensors, Wafer scale sensors, Reticule stitching, Bio- } \\
\text { medical Imaging, Mammography, DQE, CNR }\end{array}$ \\
\hline Abstract: & $\begin{array}{l}\text { Recently CMOS Active Pixels Sensors (APSs) have become a valuable } \\
\text { alternative to amorphous Silicon and Selenium Flat Panel Imagers (FPIs) in } \\
\text { bio-medical imaging applications. CMOS APSs can now be scaled up to the } \\
\text { standard } 20 \mathrm{~cm} \text { diameter wafer size by means of a reticle stitching block } \\
\text { process. However despite wafer scale CMOS APS being monolithic, sources } \\
\text { of non-uniformity of response and regional variations can persist } \\
\text { representing a significant challenge for wafer scale sensor response. Non- } \\
\text { uniformity of stitched sensors can arise from a number of factors related to } \\
\text { the manufacturing process, including variation of amplification, variation } \\
\text { between readout components, wafer defects and process variations across } \\
\text { the wafer due to manufacturing processes. This paper reports on an } \\
\text { investigation into the spatial non-uniformity and regional variations of a } \\
\text { wafer scale stitched CMOS APS. For the first time a per-pixel analysis of } \\
\text { the electro-optical performance of a wafer CMOS APS is presented, to } \\
\text { address inhomogeneity issues arising from the stitching techniques used to } \\
\text { manufacture wafer scale sensors. A complete model of the signal } \\
\text { generation in the pixel array has been provided and proved capable of } \\
\text { accounting for noise and gain variations across the pixel array. } \\
\text { This novel analysis leads to readout noise and conversion gain being } \\
\text { evaluated at pixel level, stitching block level and in regions of interest, } \\
\text { resulting in a coefficient of variation } \leq 1.9 \% \text {. The uniformity of the image } \\
\text { quality performance has been further investigated in a typical X-ray } \\
\text { application, i.e. mammography, showing a uniformity in terms of CNR } \\
\text { among the highest when compared with mammography detectors } \\
\text { commonly used in clinical practise. Finally, in order to compare the }\end{array}$ \\
\hline
\end{tabular}


2

detection capability of this novel APS with the currently used technology (i.e. FPIs), theoretical evaluation of the Detection Quantum Efficiency (DQE) at zero-frequency has been performed, resulting in a higher DQE for this detector compared to FPIs. Optical characterization, X-ray contrast measurements and theoretical DQE evaluation suggest that a trade off can be found between the need of a large imaging area and the requirement of a uniform imaging performance, making the DynAMITe large area CMOS APS suitable for a range of bio-medical applications.

\section{SCHOLARONE}




\title{
Performance of a novel wafer scale CMOS active pixel sensor for bio-medical imaging
}

\author{
M. Esposito ${ }^{1,2}$, T. Anaxagoras ${ }^{3}$, A. C. Konstantinidis ${ }^{4}$, Y. \\ Zheng $^{4}$, R. D. Speller ${ }^{4}$, P. M. Evans ${ }^{1}$, N. M. Allinson ${ }^{2}$ and \\ K. Wells ${ }^{1}$ \\ 1 Centre for Vision, Speech and Signal Processing, Faculty of Engineering and \\ Physical Sciences, University of Surrey, Guildford GU2 7XH,U.K. \\ ${ }^{2}$ School of Computer Science, University of Lincoln, Lincoln, LN6 7TS, U.K. \\ ${ }^{3}$ ISDI Ltd (Image Sensor Design and Innovation), Oxford, OX4 1YZ, U.K \\ ${ }^{4}$ Department of Medical Physics and Bioengineering, University College London, \\ WC1E 6BT, U.K. \\ E-mail: m.esposito@physics.org
}

\begin{abstract}
Recently CMOS Active Pixels Sensors (APSs) have become a valuable alternative to amorphous Silicon and Selenium Flat Panel Imagers (FPIs) in bio-medical imaging applications. CMOS APSs can now be scaled up to the standard $20 \mathrm{~cm}$ diameter wafer size by means of a reticle stitching block process. However despite wafer scale CMOS APS being monolithic, sources of non-uniformity of response and regional variations can persist representing a significant challenge for wafer scale sensor response. Non-uniformity of stitched sensors can arise from a number of factors related to the manufacturing process, including variation of amplification, variation between readout components, wafer defects and process variations across the wafer due to manufacturing processes. This paper reports on an investigation into the spatial non-uniformity and regional variations of a wafer scale stitched CMOS APS. For the first time a per-pixel analysis of the electro-optical performance of a wafer CMOS APS is presented, to address inhomogeneity issues arising from the stitching techniques used to manufacture wafer scale sensors. A complete model of the signal generation in the pixel array has been provided and proved capable of accounting for noise and gain variations across the pixel array.

This novel analysis leads to readout noise and conversion gain being evaluated at pixel level, stitching block level and in regions of interest, resulting in a coefficient of variation $\leq 1.9 \%$. The uniformity of the image quality performance has been further investigated in a typical X-ray application, i.e. mammography, showing a uniformity in terms of CNR among the highest when compared with mammography detectors commonly used in clinical practise. Finally, in order to compare the detection capability of this novel APS with the currently used technology (i.e. FPIs), theoretical evaluation of the Detection Quantum Efficiency (DQE) at zero-frequency has been performed, resulting in a higher DQE for this detector compared to FPIs. Optical characterization, X-ray contrast measurements and theoretical DQE evaluation suggest that a trade off can be found between the need of a large imaging area and the requirement of a uniform imaging performance, making the DynAMITe large area CMOS APS suitable for a range of bio-medical applications.
\end{abstract}

Keywords: CMOS Active Pixel Sensors, Wafer scale sensors, reticle stitching, Biomedical imaging, Mammography 


\section{Introduction}

Applications where large area radiation detectors are required typically use solid state detectors based on amorphous sensors (e.g. amorphous Silicon (a-Si) or amorphous Selenium (a-Se) thin film transistors (TFT)) (Rowlands and Yorkston 2000). This is especially common in medical imaging applications where a large imaging area is required ( $\geq 43 \mathrm{~cm} \times 43 \mathrm{~cm}$ for chest radiography, $25 \mathrm{~cm} \times 30 \mathrm{~cm}$ for mammography, $15-30 \mathrm{~cm}$ radius for fluoroscopy, $\geq 41 \mathrm{~cm} \times 41 \mathrm{~cm}$ for radiotherapy portal imaging), since they rely on a 1:1 aspect ratio covering the entire imaging area. Amorphous $\mathrm{Si}$ TFTs, arising from consumer-based flat-panel display technology, can easily be fabricated on a large area glass substrate to fulfill the requirements in active area even for the most demanding medical applications, e.g. chest radiography, portal imaging.

However, a-Si TFTs suffer from a number of drawbacks limiting imaging performance: high noise floor, relatively large pixel pitch, low frame rate and image lag (Weisfield and Bennett 2001, Zentai and Colbeth 2012). In fact, a-Si TFTs present a high readout noise $\left(>1000 e^{-}\right)$, due to the inherently high pixel noise combined with line noise, which tends to increase for large arrays (Colbeth 2010). Pixel pitch in a-Si devices is also physically limited by resolution and tolerance in the photolithographic techniques used, as well as by the need to have a small resistance value in the ON state for fast readout (Zentai and Colbeth 2012). Moreover amorphous devices suffer from a low frame rate, mainly due to the low electron mobility in amorphous silicon $\left(<1 \mathrm{~cm}^{2} / \mathrm{Vs}\right)$ compared to crystalline silicon ( typically $\left.1400 \mathrm{~cm}^{2} / V s\right)$, and image lag, due to abundance of charge traps in the a-Si lattice. This produces a decay time comparable to the readout speed, which needs to be compensated for, algorithmically (Siewerdsen and Jaffray 1999).

By contrast, CMOS Active Pixel Sensors (APSs)(Fossum 1995, Bigas et al. 2006, Holst and Lomheim 2011) have started to emerge as a serious alternative to FPIs in the medical imaging field, showing potential to overcome many of the limiting drawbacks of FPIs. In fact CMOS APSs are now capable of offering low noise $\left(60-150 e^{-}\right)($Arvanitis et al. 2007, Bohndiek et al. 2009, Esposito et al. 2011), as each pixel contains an active circuit (Zentai and Colbeth 2012), and a pixel pitch in the order of $25-50 \mu \mathrm{m}$, deriving from higher resolution lithographic processes and higher levels of integration reached in the CMOS manufacturing processing, technically and economically driven by integrated circuit applications (Lo 1998).

APSs also offer a higher frame rate, based on the higher charge mobility of crystalline silicon compared to a-Si, true random access via column parallel readout and an absence of image artifacts (e.g. image lag). These advantages, together with low power consumption, decreasing proportionally to the minimum feature size in the specific technology used (Agranov et al. 2005), and potential for a low cost and fast scaling technology based on standard consumer-based CMOS fabrication techniques, have made CMOS APSs, a valuable alternative in the bio-medical imaging field.

Furthermore, APSs are based on the use of in-pixel active circuits, which can then be designed to program operations at the pixel level. On-chip intelligence for APSs can be developed down to the pixel level to implement counting functions, rudimentary image processing, selective readout of Region-of-Interest (ROI) and readout of small ROIs with position triggered by an external event. On-chip intelligence also has the potential to implement a number of readout modalities which can improve imaging tasks, such as on-line dose sensing (Korthout et al. 2009) to minimize patient dose, and dynamic tracking of specific imaging feature (i.e. fiducial markers in radiotherapy 
Table 1. Summary of the design specifications and optical performance for CMOS large area detectors.

\begin{tabular}{|c|c|c|c|c|c|c|}
\hline & $\begin{array}{l}\text { Tile } \\
\text { Area } \\
\left(\mathrm{cm}^{2}\right)\end{array}$ & $\begin{array}{l}\text { Side } \\
\text { Buttable }\end{array}$ & $\begin{array}{l}\text { Pixel } \\
\text { size } \\
(\mu \mathrm{m})\end{array}$ & $\begin{array}{l}\text { Frame } \\
\text { rate } \\
(\text { fps })\end{array}$ & $\begin{array}{l}\text { Noise } \\
\text { floor } \\
\left(\mathrm{e}^{-}\right)\end{array}$ & $\begin{array}{l}\text { Conversion } \\
\text { gain }\end{array}$ \\
\hline $\begin{array}{l}\text { RedEye1 } \\
\text { (RadIcon 2013) }\end{array}$ & $2.46 \times 4.92$ & 3 & 48 & 4.5 & 150 & \\
\hline $\begin{array}{l}\text { C97732 DK-11 } \\
\text { (Hamamatsu 2013) }\end{array}$ & $12 \times 12$ & - & 50 & 1 & 1250 & - \\
\hline $\begin{array}{l}\text { CMOS APS } \\
\text { (Korthout et al. 2009) }\end{array}$ & $7.73 \times 14.5$ & 3 & 33.55 & 8.7 & 175 & $2.45 \mu \mathrm{V} / \mathrm{e}^{-}$ \\
\hline $\begin{array}{l}\text { VLA CMOS } \\
\text { (Farrier et al. 2009) }\end{array}$ & $4.9 \times 9.8$ & 3 & 96 & 1.3 & 250 & $0.21 \mu \mathrm{V} / \mathrm{e}^{-}$ \\
\hline $\begin{array}{l}\text { CMOS APS } \\
\text { (Ay and Fossum 2006) }\end{array}$ & $7.37 \times 7.75$ & - & 18 & 1.25 & 240 & $2.27 \mu \mathrm{V} / \mathrm{e}^{-}$ \\
\hline $\begin{array}{l}\text { LAS } \\
\text { (Bohndiek et al. 2009) } \\
\text { (Konstantinidis et al. 2010) }\end{array}$ & $5.6 \times 5.6$ & - & 40 & 20 & 50 & $4.6 \mathrm{e}^{-} / \mathrm{DN}$ \\
\hline $\begin{array}{l}\text { CMOS APS } \\
\text { (Reshef et al. 2009) }\end{array}$ & $12 \times 15$ & 3 & 150 & 30 & - & - \\
\hline $\begin{array}{l}\text { CMOS APS } 2923 \\
\text { (Konstantinidis et al. 2012) }\end{array}$ & $11.5 \times 14.5$ & 2 & 75 & 26 & $\begin{array}{l}361.9^{a} \\
164.9^{b}\end{array}$ & $\begin{array}{l}119.6 \mathrm{e}^{-} / \mathrm{DN}^{a} \\
38.8 \mathrm{e}^{-} / \mathrm{DN}^{b}\end{array}$ \\
\hline
\end{tabular}

${ }^{a}$ High Full Well

${ }^{b}$ Low Full Well

(Osmond et al. 2008)).

Recently, developments in photolithography have made available the realization of large area devices integrated onto a silicon wafer to create a contiguous sensor array. The associated technique, referred to as a stitching process (Scheffer 2007, Turchetta et al. 2011), is based on the use of a mask reticle, comprising several functional blocks or a larger circuit (e.g. pixels and readout electronics), which is stepped and repeated, in whole or in part, across a silicon wafer to create modularly different sectors of a large circuit, so that sensors of arbitrary size can be manufactured, limited only by wafer size. As a result of this, a number of large area CMOS APSs is currently available and listed in Table 1, together with design specifications and optical performance.

However, sources of non-uniformity of response and regional variations can affect wafer scale sensors, representing a significant challenge for these detectors. Nonuniformity of stitched sensors can arise from a number of factors related to the manufacturing process, including lack of refocussing of the stepper at each reticle position, variations of the amplification, variation between readout components, wafer defects and variations across the wafer, such as threshold voltage, leakage current, substrate doping, mobility, trans-conductance, etc.

Non-uniformity in large area sensors can represent a significant drawback as regional differences in noise and conversion gain can generate local changes in imaging performance, such as Signal and Contrast-to-Noise Ratio, introducing anisotropy in image resolution. They also produce uncertainty when the sensor is calibrated using parameters derived globally on the whole sensor area, ultimately limiting the sensor 


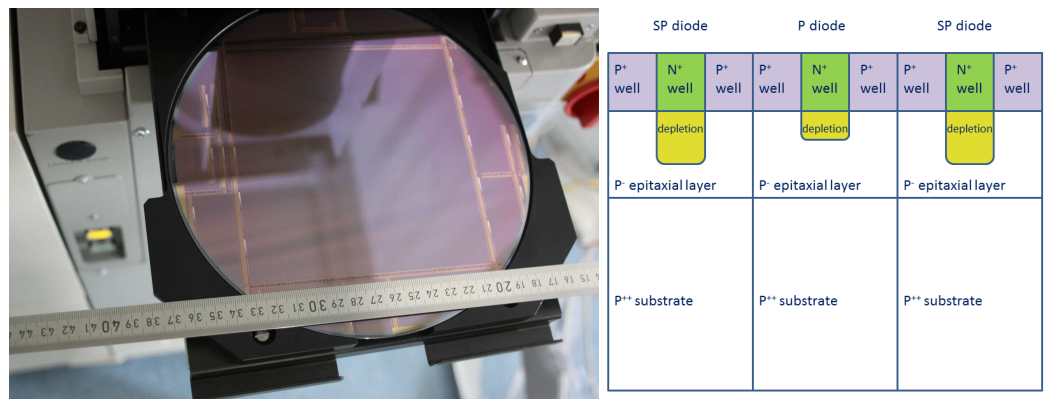

(a)

(b)

Figure 1. a) The DynAMITe chip wafer. The boundaries of the chip region are visible with the ruler denoting a $12.8 \mathrm{~cm}$ edge dimension. b) A schematic representation of the diodes arrangement for the DynAMITe detector. Sub-pixels show a deeper depletion width compared to Pixels.

resolution. Finally, large variations in noise and conversion gain can entail a decrease in dynamic range.

Non-uniformity and regional variations in large area stitched sensors have been investigated by some groups recently. Zin et al. (Zin et al. 2010) investigated spatial non-uniformity of a Large Area Sensor (LAS) (Bohndiek et al. 2009), (Konstantinidis et al. 2010). Sensor non-uniformity was evaluated in each of the stitched regions of the sensor resulting in variations between $3.85-5.67$ and $3.79-7.02 \%$ for read noise and gain respectively.

In this paper, we report an investigation into the spatial non-uniformity of a wafer scale stitched CMOS APS. Key metrics for sensor performance, such as read noise, conversion gain and full well capacity have been optically characterized for the whole sensor by means of the Photon Transfer Curve (PTC) technique. Nevertheless when optical characterization involves wafer scale sensors, where manufacturing techniques are based on regional processes (i.e. reticle stitching), evaluating averaged parameters in specific regions of the sensor can be misleading. To overcome these limitations, a per-pixel PTC analysis of the sensor was performed, analyzing the results on a per pixel basis and at a stitching block level, providing a more detailed understanding of the uniformity of response of the sensor. Furthermore the Fixed Pattern Noise (FPN) has been evaluated at different grouping levels across the entire sensors array and for each stitched region. Finally uniformity and regional variations of this large area CMOS sensor have been evaluated with X-rays for a common mammographic set-up. The remainder of this paper is organized as follows: Section 2 describes the design of the DynAMITe wafer scale sensor used in this work and the techniques for electrooptical and X-ray assessment of the sensor performance; Section 3 reports on the electro-optical parameters of the DynAMITe sensor evaluated at pixel level, reticlelevel and in region of interest. A further stage of this non-uniformity investigation (Section 3.5) involved evaluation of Contrast-to-Noise Ratio (CNR) in mammographic images and comparison with commercially available mammography detectors. Finally, theoretical evaluation of Detective Quantum Efficiency for APSs, in comparison with FPIs, is reported in Section 3.6, followed by conclusions in Section 4. 


\section{Materials and Methods}

\subsection{The DynAMITe detector}

The DynAMITe CMOS APS is fabricated in a standard $0.18 \mu \mathrm{m}$ CMOS technology using reticle stitching (Scheffer 2007), covering a total active area of $12.8 \times 13.1 \mathrm{~cm}^{2}$. It is also designed to be two-side buttable so that the imaging area can be further increased up to $25.6 \times 26.2 \mathrm{~cm}^{2}$. A picture of the silicon wafer from which the DynAMITe sensor has been diced is shown in Figure 1 a) .

The novel concept underlying the development of this detector lies in simultaneously using high and low well capacity diodes in the same pixel array. High well capacity diodes are able to provide high dynamic range, whereas low well capacity diodes can offer low noise, offering an extended dynamic range when these are combined. This sensor thus consists of two grids of different well capacity diodes, which are geometrically super-imposed. Large well capacity diodes, referred to as Pixel (P) diodes are placed on a $1280 \times 1312$ pixel matrix with $100 \mu \mathrm{m}$ pitch, whereas low well capacity diodes, referred to as Sub-Pixel (SP) diodes are arranged on a $2560 \times 2624$ pixel grid with $50 \mu \mathrm{m}$ pitch. SP diodes are reset at a higher reset voltage than $\mathrm{P}$ diodes, leading to the creation of different depletion widths (Figure $1 b)$ ). The deeper depletion width of SP type diodes allows, as radiation interacts with the sensor, the first generated charge to be collected by such low noise pixels. Hence low intensity signals are detected with an intrinsic low noise, resulting in in a good Signal-to-Noise Ratio (SNR) performance. As Sub-Pixels reach near saturation, then P type diodes start collecting providing an extended dynamic range. The intrinsic higher noise of those pixels does not degrade the SNR performance of the sensor, as the higher noise level has to be compared with a higher intensity signal.

Readout of CMOS arrays is based on a rolling readout technique: all pixels in a row are reset and readout in parallel, but different rows are processed sequentially (Fish and Yadid-Pecht 2004). The readout architecture of the DynAMITe sensor is based on a dual readout chain: each pixel is connected to two reset, select and output lines. Doubling the readout electronics allows either to increase the readout speed or to readout different sensor regions or pixel types in combination.

An increase in readout speed is achieved when the whole pixel array or a Region of Interest (ROI) is readout by using both readout chains. In fact, the two separate electronic chains can be synchronized in a ping-pong arrangement where two rows are readout simultaneously, one by each chain. This arrangement effectively reduces the number of rows/pixels readout by each chain to a half, thus increasing the readout speed of a factor of two.

By using the ping-pong architecture, the maximum readout frame rateł for the $1280 \times 1312 \mathrm{P}$ type matrix is designed for $90 \mathrm{fps}$ whereas this figure is $30 \mathrm{fps}$ for the $2560 \times 2624$ SP type matrix, while the use of ROIs allows for a faster readout, with frame rate proportional to the ROI size. As the dual readout chain can be used either

$\ddagger$ The maximum frame rate is the reciprocal of the minimum integration time of the sensor, i.e. the time needed to readout all the pixels. This is defined as

$$
T=\left(N_{\text {rows }} \times T_{\text {row }}\right)+\left(\frac{N_{\text {pixels }}}{N_{\text {amp }}} \times T_{\text {clock }}\right)
$$

where $N_{\text {rows }}$ is the number of rows to readout, $T_{\text {row }}$ is the time required to sample a row, $N_{\text {pixels }}$ is the number of pixels in the array, $N_{a m p}$ is the number of amplifiers and $T_{\text {clock }}$ is the time required to clock a pixel voltage towards the amplifiers. 
in the ping-pong architecture or operated independently, several readout modalities are implemented for this sensor:

- Pixel full matrix (90 fps);

- Sub-Pixel full matrix (30 fps);

- Pixel full matrix (45 fps) readout simultaneously with Sub-Pixel full matrix (15 fps);

- Pixel full matrix (45 fps) readout simultaneously with Pixel ROI (714 fps for $100 \times 100 \S$ pixel ROI);

- Sub-Pixel full matrix (15 fps) readout simultaneously with Sub-Pixel ROI (714 fps for $100 \times 100$ pixel ROI);

- Pixel full matrix (45 fps) readout simultaneously with Sub-Pixel ROI (714 fps for $100 \times 100$ pixel ROI);

- Sub-Pixel full matrix (15 fps) readout simultaneously with Pixel ROI (714 fps for $100 \times 100$ pixel ROI).

Additionally by using the above readout modalities it is possible to perform Non Destructive Readout (Esposito et al. 2011), where the full frame of the entire pixel matrix can be readout at a given frame rate using one electronic chain, while an ROI is simultaneously readout at a higher frame rate using the second chain. As the ROI is not reset, during readout, collected charge is accumulated until the full matrix is readout. Non Destructive Readout allows for Correlated Double Sampling (CDS) (El Gamal and Eltoukhy 2005) and online dose sensing.

The DynAMITe sensor has also been designed according to the radiation hardness-by-design methodology (Lacoe 2008). In fact all the in-pixel transistors have been designed with source and drain physically enclosed according to the Enclosed Layout Geometry and $\mathrm{P}+$ doped guard rings have been added in each pixels, in order to reduce radiation induced edge and inter-device leakage current, respectively. The sensor has been tested with $160 \mathrm{kVp} \mathrm{X-rays} \mathrm{and} \mathrm{proved} \mathrm{to} \mathrm{be} \mathrm{radiation} \mathrm{tolerant} \mathrm{up} \mathrm{to}$ a dose of $94 \mathrm{kGy}(\mathrm{Si})$ (Esposito et al. 2012).

\subsection{Stitching}

A schematic of the stitching block process used for the DynAMITe sensor is reported in Figure 2, showing the 8 types (labelled as A-I regions) of sub-block mask used for manufacture. The sensor imaging area has been constructed by stepping a sub-block mask of $18.0 \mathrm{~mm} \times 25.6 \mathrm{~mm}$ across the wafer (E block), creating a $180 \times 256$ pixel block and a $360 \times 512$ pixel block for Pixels and Sub-Pixels respectively. Additional blocks are placed at the edges of the sensor:

- 1 A-type block and 5 D-type blocks for row addressing;

- 1 G-type block for the Grey Code Counter which drives the row addressing;

- 7 H-type blocks and 1 I-type block for the outputs;

- 7 B-type $(18 \mathrm{~mm} \times 3.2 \mathrm{~mm}), 1 \mathrm{C}$-type $(2 \mathrm{~mm} \times 3.2 \mathrm{~mm})$ and 5 F-type blocks $(2$ $\mathrm{mm} \times 25.6 \mathrm{~mm})$ as further imaging area to reduce dead space at the edge in the 2 -side buttable configuration.

$\S$ A $100 \times 100$ ROI is presented in this example. However ROI size can be chosen arbitrary, leading to different frame rates proportional to the ROI size. 


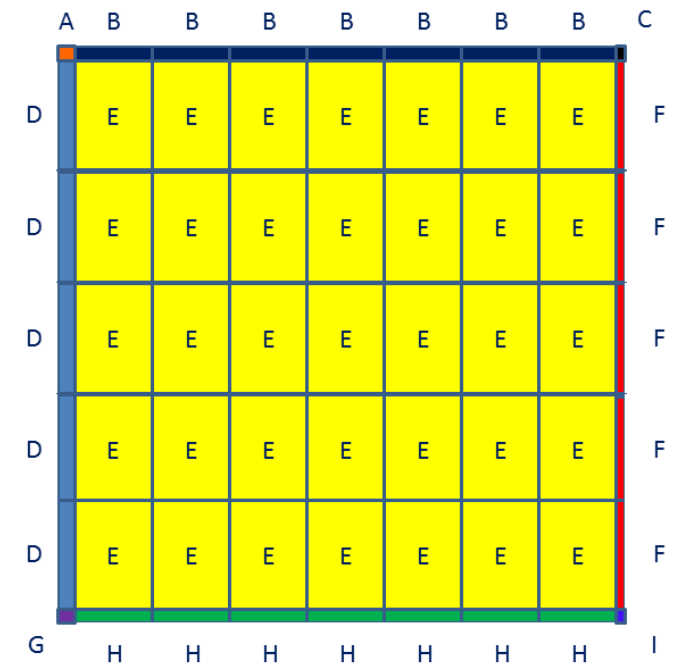

Figure 2. Schematic of the stitching block process used for the DynAMITe sensor. Eight types of sub-block mask are reported: blocks E, B, C, F which contribute to the sensor active area, blocks $\mathrm{A}$ and $\mathrm{D}$ for row addressing, block $\mathrm{G}$ for the Grey code counter, blocks $\mathrm{H}$ and I for the outputs.
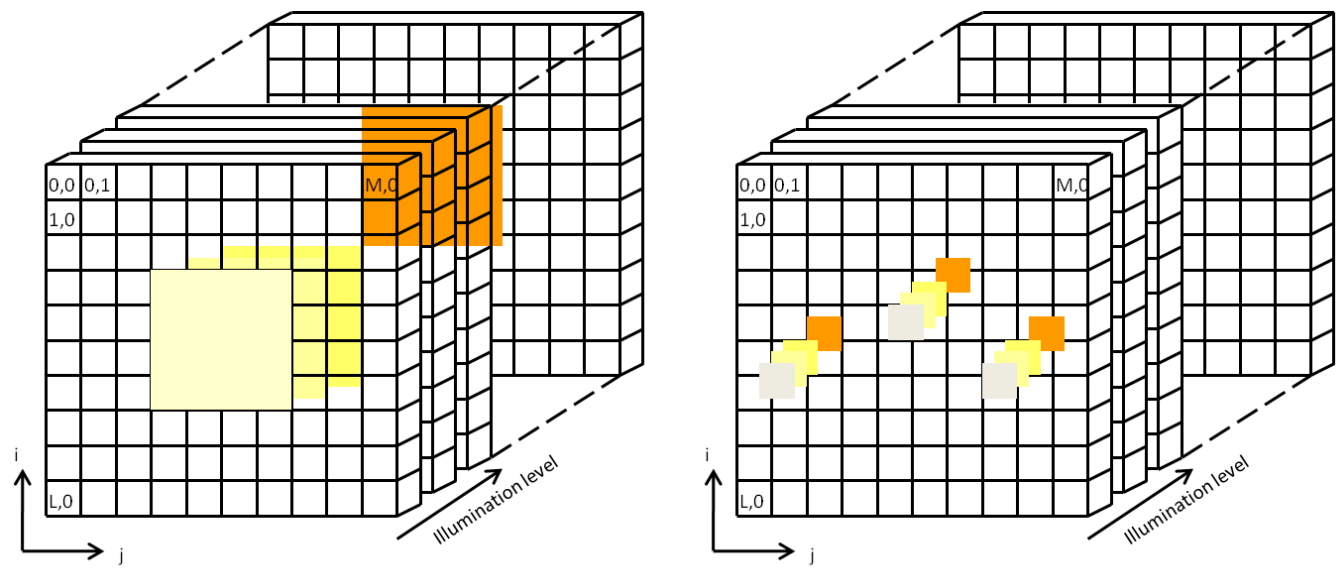

Figure 3. Schematic comparison between a Photon Transfer Curve generated by averaging noise and signal in a region of interest (left) and by evaluating it as a per-pixel function (right). Index $i$ refers to row, index $j$ to columns and $k$ to the progressively increasing illumination levels.

E blocks are repeated five times in the Y-axis direction and seven times in the $\mathrm{X}$ axis direction, realizing an imaging area of $1260 \times 1280$ P-type pixels and $2520 \times 2560$ SP-type pixels which increases up to $1280 \times 1312$ and of $2560 \times 2624$ pixels respectively when $\mathrm{B}, \mathrm{C}$ and $\mathrm{F}$-type blocks are taken into account. 
Figure 4. Schematic representation of the signal generated by $P$ photons, as described in Equation (1).

\subsection{Photon Transfer curve}

Assessment of the sensor performance in terms of image noise and signal is an essential step to optimize imaging sensors and evaluate their reliability in specific applications. Noise sources in APSs can be classified into two different types: temporal noise, i.e. having temporal dependence, and spatial noise, due to noise sources which vary across the detector matrix. Read and shot noise belong in the first category, the first being due to signal-independent noise sources (e.g. reset noise, off-chip and on-chip amplifier noise, quantization noise, dark current shot noise), the latter depends on the detected signal (Poisson statistics of interacting radiation quanta). FPN is considered a spatial related source of noise, as it is ascribable to non-uniformities in the manufacturing process and to differences in pixel and column voltages as well as variations in column amplifiers.

The Photon Transfer Curve (Stark et al. 1992, Beecken and Fossum 1996, EMVA 2005, Janesick 2001) represents the standard for evaluating performance parameters, such as read noise, conversion gain and full well capacity, and provides means to isolate and quantify noise components in the sensor response.

The signal generated in a pixel by $P$ incident photons, expressed in digital numbers $(\mathrm{DN}) \|$, can be written as

$$
S(D N)=P Q E \eta_{i} S_{v} A_{I N T} A_{E X T} A_{A D C}
$$

where $Q E$ is the interacting efficiency (interacting photons/incident photons), $\eta_{i}$ is the quantum yield (number of $e^{-}$generated per interacting photon), $S_{v}$ is the sensitivity of the sense node $\left(\mathrm{V} / e^{-}\right), A_{I N T}$ is the gain of the in-pixel amplifier $(\mathrm{V} / \mathrm{V}), A_{E X T}$ is the gain of the external amplifier $(\mathrm{V} / \mathrm{V})$ and $A_{A D C}$ is the gain of the ADC (DN/V). A schematic representation of the signal generation process is shown in Figure 4. The conversion gain can be defined as

$$
K\left(e^{-} / D N\right)=\frac{1}{S_{v} A_{I N T} A_{E X T} A_{A D C}}
$$

For incident photon of wavelength $\lambda>400 \mathrm{~nm}$, a single electron-hole pair is generated per interacting photons, i.e. $\eta_{i}=1$. Using this and Equation (2), Equation (1) can be re-written as

$$
S(D N)=\frac{P_{I}}{K\left(e^{-} / D N\right)}
$$

\| This is the pixel voltage converted into a digital value by sensor ADCs. 
The conversion gain $K$ can then be evaluated by relating it to the signal variance $\sigma_{S}^{2}$. The variance of Equation (3) can be found by applying the propagation of the errors formula, and by adding the read noise $\sigma_{R}^{2}(D N)$ in quadrature

$$
\sigma_{S}^{2}(D N)=\left(\frac{\partial S}{\partial P_{I}}\right)^{2} \sigma_{P_{I}}^{2}+\left(\frac{\partial S}{\partial K}\right)^{2} \sigma_{K}^{2}+\sigma_{R}^{2}(D N)
$$

Using the assumption of a Poisson distribution for the interacting photons $\left(\sigma_{P_{I}}^{2}=P_{I}\right)$ and of a negligible variance for the conversion gain $\left(\sigma_{K}^{2} \sim 0\right)$, Equation $(4)$ becomes

$$
\sigma_{S}^{2}(D N)=\frac{P_{I}}{K^{2}}+\sigma_{R}^{2}(D N)
$$

Substituting Equation (3) into Equation (5), the conversion gain can be expressed as follows

$$
K=\frac{S(D N)}{\sigma_{S}^{2}(D N)-\sigma_{R}^{2}(D N)}
$$

where $\sqrt{\sigma_{S}^{2}(D N)-\sigma_{R}^{2}(D N}$ is the signal shot noise $\sigma_{\text {shot }}$. Plotting logarithmically the signal noise $\sigma_{S}$ of the sensor as a function of the mean signal $S$ gives the PTC. In order to calculate the conversion gain $K\left(e^{-} / D N\right)$, it is necessary to extract the signal shot noise from the total sensor noise. Temporal invariant components can be removed from the signal noise subtracting two consecutive frames which will yield a FPN suppression. The read noise can be suppressed by subtracting the variance of a differenced dark frame $\left(P_{I}=0\right)$ from the signal variance $\sigma_{S}^{2}$. The PTC data are generated exposing the sensor to different illumination levels from dark to saturation, provided by an LED array centred at $\lambda=523 \mathrm{~nm}$ (bandwidth $35 \mathrm{~nm}$ ) coupled to a lens and a single neutral density filter, to achieve uniform illumination. A calibrated photodiode was also used to estimate the photon flux at the sensor position. The rms sensor noise was then evaluated as a function of the sensor signal. The gain of the system is determined from the noise measurements, under assumption of Poisson distributed input signal noise. Conventionally, both noise and signal are calculated as an average in an ROI $(512 \times 512$ Sub-Pixel and $256 \times 256$ Pixel ROI in this work). Hence this procedure provides an average information on the behavior of the sensor.

\subsection{Per-pixel Photon Transfer Curve}

In order to gain information on the uniformity of response and regional variations of the sensor, the Photon Transfer Curve is evaluated on a per pixel basis from generalizing Equation (6) into:

$$
K(i, j)=\frac{S(D N)(i, j)}{\sigma_{S}^{2}(D N)(i, j)-\sigma_{R}^{2}(D N)(i, j)}
$$

representing a relation between the pixel signal $S(i, j)$ at location $(\mathrm{i}, \mathrm{j})$ and the associated pixel signal noise $\sigma_{S}(i, j)$, resulting in the evaluation of a per-pixel conversion gain $K(i, j)$ and a read noise $\sigma_{R}(i, j)$ matrix. A schematic to highlight the differences between ROI resulting from ROI average and the per-pixel procedure is shown in Figure 3. 


\subsection{Fixed Pattern Noise}

Fixed Pattern Noise (FPN) represents the spatial variation of the output image of a sensor due to different gains and offsets in pixel transistors and to column amplifiers. FPN can be analysed considering separately the effect due to pixels and columns (El Gamal et al. 1998):

$$
F_{i, j}=X_{i, j}+Y_{j}
$$

where $X_{i, j}$ is the pixel-to-pixel(P-P) FPN due to gain and offset variation in pixel transistors and $Y_{j}$ is the column-to-column (C-C) FPN due to variation in column amplifiers of the column parallel readout. The two terms contributing to the FPN can be calculated by evaluating the difference of each pixel signal $\overline{S_{i, j}}$, under uniform illumination, averaged over $N$ frames, and the overall average signal $\bar{S}$ given by

$$
\bar{S}=\frac{\sum_{i, j, m}^{L, M, N} S_{i, j, m}}{L M N}
$$

where $i, j, m$ are rows, columns and frames indices and $L, M, N$ are their maximum values. The components of the FPN $\left(\overline{Y_{j}}\right.$ and $\left.\overline{X_{i, j}}\right)$ and their variances $\left(\overline{\sigma_{Y}^{2}}\right.$ and $\left.\overline{\sigma_{x}^{2}}\right)$ are calculated as follows

$$
\begin{aligned}
& \overline{Y_{j}}=\frac{\sum_{i=1}^{L} F_{i, j}}{L} \\
& \overline{X_{i, j}}=F_{i, j}-\overline{Y_{j}} \\
& \overline{\sigma_{Y}^{2}}=\frac{\sum_{j=1}^{M} \bar{Y}_{j}^{2}}{M-1} \\
& \overline{\sigma_{X}^{2}}=\frac{\sum_{i=1}^{L} \sum_{j=1}^{M}{\overline{X_{i, j}}}^{2}}{M(L-1)}
\end{aligned}
$$

Column-to-column $\left(\overline{\sigma_{Y}^{2}}\right)$ and pixel-to-pixel FPN $\left(\overline{\sigma_{X}^{2}}\right)$ metrics are conventionally expressed in terms of the percentage of the average signal $\bar{S}$ for a given illumination level, which is $50 \%$ of saturation in this work.

\subsection{Contrast-to-Noise Ratio}

The uniformity of the image quality performance, deriving from the electro-optical properties listed in the previous sections, have been investigated to facilitate understanding of detector performance in clinical routine applications.

$\mathrm{X}$-ray measurements in a common mammography set-up were performed to evaluate the uniformity of the CNR across the pixel array.

A $25 \mathrm{kVp}$ X-ray source with Mo anode was used together with a $30 \mu \mathrm{m}$ Mo filtration. A CsI scintillator, $150 \mu \mathrm{m}$ thick, was chosen to allow photon conversion before detection and this was coupled to a $3 \mathrm{~mm}$ thick Fiber Optic Plate (FOP). A test object of $45 \mathrm{~mm} \times 25 \mathrm{~mm} \times 120 \mathrm{~mm}$, made of Perspex, was used to assess the CNR in the stitching blocks. The object was placed above the detector with the $45 \mathrm{~mm}$ 
side parallel to the beam axis, in order to deliver a breast-equivalent thickness of 53 $\mathrm{mm}$ (Dance et al. 2012). Since the object only covers $20 \%$ of the detector area (its projection on the detector area is $25 \mathrm{~mm} \times 120 \mathrm{~mm}$ ), it was stepped across the detector reticle and images acquired at each step.

Signal within the test object $\left(S_{o}\right)$ and within the background $\left(S_{b}\right)$ were evaluated in ROIs corresponding to the detector stitching block, together with their standard deviation ( $\sigma_{o}$ and $\sigma_{b}$ respectively), to derive CNR:

$$
C N R=\frac{S_{o}-S_{b}}{\sqrt{\sigma_{o}^{2}+\sigma_{b}^{2}}}
$$

In order to assess the uniformity of the CNR across the detector area, a figure of merit $\left(C N R_{u n i}\right)$ was evaluate following (Baldelli et al. 2009):

$$
C N R_{\text {uni }}=\frac{N\left(\Delta_{15 \%-C N R_{\text {mean }}}\right)}{N_{\text {total }}} \%
$$

as the ration between the number of ROIs where CNR is within $15 \%$ of the mean CNR $\left(C N R_{\text {mean }}\right)$ and the total number of ROIs used for the study.

\subsection{Detective Quantum Efficiency}

Detective Quantum Efficiency (DQE) is commonly used method to characterize imaging performance, describing the Signal-to-Noise ratio through the imaging chain (Metz et al. 1995). DQE is a frequency-dependent figure, however many of the signal and noise transfer properties can be studied at zero-frequency $(\mathrm{DQE}(0))$.

A cascaded linear system can be used to model signal and noise properties of imaging detectors (Siewerdsen et al. 1997). This approach is based on describing the imaging system as a series of discrete stages, each of which represents a quantum gain or blurring process. For a gain stage, the mean fluence output quanta $\bar{q}_{i}$ can be related to the input quanta $\bar{q}_{i-1}$ by means of the mean gain $\bar{g}_{i}$ at that stage $i$ :

$$
\bar{q}_{i}=\bar{g}_{i} \bar{q}_{i-1}
$$

For an imaging system based on indirect conversion, the first stage of the cascaded linear system is represented by the interaction of X-ray incident quanta in the converter. The gain at this stage is given by

$$
\bar{g}_{1}=\frac{\int_{0}^{E_{M A X}} q_{0}(E)\left(1-e^{-\mu(E) T}\right) \mathrm{d} E}{\int_{0}^{E_{M A X}} q_{0}(E) \mathrm{d} E}
$$

where $\mu(E)$ is the attenuation coefficient at the energy $\mathrm{E}$ and $\mathrm{T}$ is the thickness of the converter. The generation and emission of optical quanta in the converter are represented by the gain $\bar{g}_{2}$, comprising two terms $\bar{g}_{2 a}$ and $\bar{g}_{2 b}$. The first term represents the average number of optical photons generated per interacting X-ray:

$$
\bar{g}_{2 a}=\frac{\int_{0}^{E_{M A X}} q_{1}(E) \bar{g}_{2 a}(E) \mathrm{d} E}{\int_{0}^{E_{M A X}} q_{1}(E) \mathrm{d} E}
$$

where $\bar{q}_{1}(E)$ is the spectrum of the interacting photons and $\bar{g}_{2 a}(E)$ is the mean number of optical photons generated per X-ray of energy E. The second term contributing to 


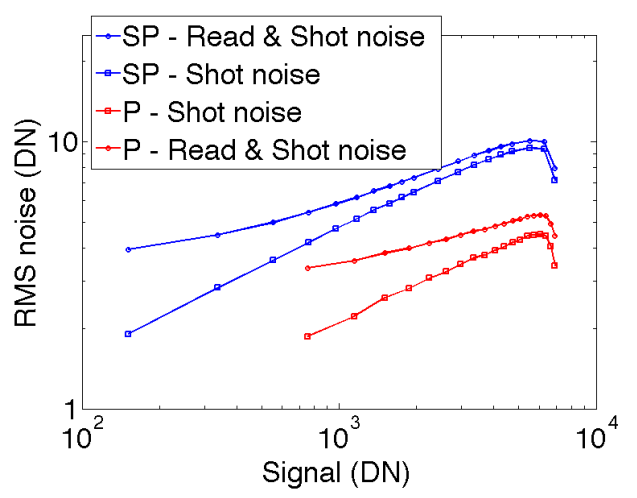

(a)

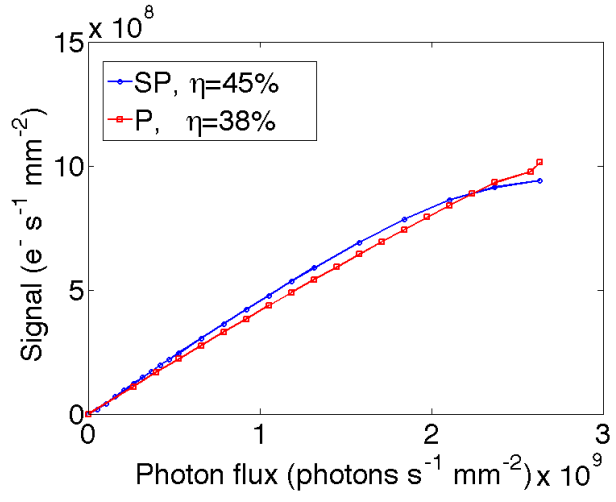

(b)

Figure 5. a) Read and shot noise PTCs displayed with the decomposed shot noise component evaluated in a region of interest for Sub-Pixels and Pixels. b) Linearity curves for Sub-Pixels and Pixels calculated in an ROI. The number of electrons produced in the pixel for a given number of incident photons is plotted as function of the photon flux.

the gain at the converter stage, $\bar{g}_{2 b}$, is the fraction of generated optical photons which will exit the converter. Gain at the converter stage has a variance, or Poisson excess $\epsilon_{g 2}$ (Cunningham et al. 1994), related to the Swank noise (Swank 1973). Values of the Swank noise reported in (Zhao et al. 2004) are used in this work. The final gain stage is represented by the fraction of optical quanta detected. Following (Siewerdsen et al. 1997), this factor $\left(\bar{g}_{4}\right)$ is assumed to be equivalent to the fill factor.

This cascaded model allows prediction of the theoretical $\mathrm{DQE}(0)$ for imaging systems, once properties of the scintillator system and detector parameters (i.e. read noise, pixel pitch and fill factor) are known. The DQE(0) can be expressed as

$$
D Q E(0)=\frac{\bar{g}_{1} \bar{g}_{2} \bar{g}_{4}}{1+\bar{g}_{4}\left(\bar{g}_{2}+\epsilon_{g_{2}}\right)+\frac{\sigma_{R}^{2}}{a_{\text {pixel }}^{2} \bar{q}_{0} \bar{g}_{1} \bar{g}_{2} \bar{g}_{4}}}
$$

Theoretical prediction of $\mathrm{DQE}(0)$ is of great importance for this work, as it allows comparison with detection performance of the detector under study with conventional FPIs, routinely used in X-ray imaging applications.

\section{Results and Discussion}

\subsection{Photon Transfer Curve}

Figure 5 displays the PTCs for Sub-Pixels and Pixels calculated from averaged noise and signal in an ROI (see figure 3). The noise components are decomposed into "read and shot noise" and "shot noise" after FPN subtraction. The curves for signal shot noise, plotted logarithmically, as a function of the mean output signal, provide the read noise and full well capacity of the sensor. The read noise and full well capacity can then be converted from relative digital numbers to absolute units of electrons after deriving the conversion gain of both pixel arrays, as described in Section 2.3. The conversion gain is then calculated using the slope of these curves in their respective linear region. Dynamic range can be calculated from these parameters as 
A novel wafer scale CMOS active pixel sensor for bio-medical imaging

Table 2. Summary of the performance parameters of the Pixel and Sub-Pixel arrays calculated for a region of interest.

\begin{tabular}{lll}
\hline Parameter & Pixels & Sub-Pixels \\
\hline Conversion gain $\left(e^{-} / D N\right)$ & $293.3 \pm 0.8$ & $50.0 \pm 0.2$ \\
Read noise $\left(e^{-}\right)$ & $780 \pm 1$ & $149.9 \pm 0.7$ \\
Full well capacity $\left(e^{-}\right)$ & $1.9 \pm 0.7 \times 10^{6}$ & $0.3 \pm 0.2 \times 10^{6}$ \\
Quantum efficiency $\eta(\%)$ & 38 & 45 \\
Dynamic range (dB) & 68 & 66 \\
INL (\%) & 2 & 0.4 \\
\hline
\end{tabular}

$D R=20 \log _{10} \frac{F W C}{\sigma_{R}}$, while Integral Non-Linearity (INL) is the difference between the data points and the linear regression fit $(\Delta)$ in the linearity plot (Figure $5 b)$ ), computed as $I N L=\left(\Delta_{\max }-\Delta_{\min }\right) / A D C_{\text {fullscale }} \times 100$.

The derived electro-optical performance parameters calculated from the PTC for Sub-Pixel and Pixel arrays are reported in Table 2. The linearity curve for both pixel arrays, displayed in Figure $5 \mathrm{~b}$ ), shows the result of using the conversion gain to calculate the number of signal electrons as a function of the number of incident photons. The slope of these curves yields a quantum efficiency $\eta$ of $45 \%$ for the Sub-Pixel array and $38 \%$ for the Pixel array $(\lambda=523 \mathrm{~nm})$. This can be explained when considering the diode area inside both pixel arrays, resulting in a larger sensitive area for Sub-Pixels compared to Pixels.

\subsection{Per-pixel Photon Transfer Curve}

Conversion gain and read noise have been calculated as a per-pixel function, using Equation (7). A conversion gain and read noise value of zero has been assigned to Pixels and Sub-Pixels corresponding to a determination coefficient $\left(r^{2}\right)$ lower than 0.5 , i.e. poor fit quality, in the linear fit of Equation (7), (less than $1 \%$ of the pixel array). The conversion gain matrices for Pixels and Sub-Pixels are shown inFigure 6 inset $a$ ) and $f$ ) respectively together with the relevant histograms (inset $b$ ) and $f$ )), whereas the read noise of Pixels and Sub-Pixels is displayed in Figure 6 inset $c$ ) and g) respectively together with the relevant histograms (inset $e$ ) and $h$ )).

The color scales represent the conversion gain per-pixel in $e^{-} / D N$ (Figure 6 (a) and $c)$ ))) and the read noise expressed in $e^{-}$(Figure $6(e)$ and $\left.g\right)$ )). Matrices for conversion gain and read noise (Figure 6) are devoid of specific patterns of variation across the entire imaging area, suggesting the stitching procedure in sensor fabrication did not introduce variations at reticle level or at the reticle edges.

Additionally data reported for the Sub-Pixel array present a group of pixels with significant deviation from the average values between column 83 and column 96, distinguishable by a vertical dashed line in Figure $6 \mathrm{c}$ ),g). This is due to readout issues at the amplifier level for the specific sensor under characterization.

Histograms within Figure 6 have been fitted with a log-normal probability density function $f(x)$

$$
f(x)=\frac{1}{x \cdot \sigma \sqrt{2 \pi}} \exp \left\{\frac{-1}{2 \sigma^{2}}(\log (x)-\mu)^{2}\right\}, x>0
$$

where $\mu$ and $\sigma$ represent the mean and the standard deviation of the log-normal distributed variable. The log-normal fit for all distributions of Figure 6 gave a 
Table 3. Results of the log-normal fit for read noise and conversion gain distributions in term of mean $\mu$ and standard deviation $\sigma$

\begin{tabular}{llllll}
\hline Parameter & Distribution & Symbol & Unit & Pixels & Sub-Pixels \\
\hline$\mu$ & Gain & $K$ & $\mathrm{e}^{-} / \mathrm{DN}$ & $296 \pm 1$ & $59 \pm 1$ \\
$\sigma$ & Gain & $K$ & $\mathrm{e}^{-/ D N}$ & $90.14 \pm 0.08$ & $20.94 \pm 0.02$ \\
$\sigma / \mu \times 100$ & Gain & $K$ & $\%$ & 50 & 64 \\
$\mu$ & Noise & $\sigma_{R}$ & $\mathrm{e}^{-}$ & $887 \pm 1$ & $262 \pm 1$ \\
$\sigma$ & Noise & $\sigma_{R}$ & $\mathrm{e}^{-}$ & $451.75 \pm 0.07$ & $168.14 \pm 0.01$ \\
$\sigma / \mu \times 100$ & Noise & $\sigma_{R}$ & $\%$ & 30 & 35 \\
\hline
\end{tabular}

coefficient of determination $\mathbf{R}^{2}>0.99$. Mean $(\mu)$ and standard deviation $(\sigma)$ have also been evaluated and results are reported in Table 3 with the fitting errors. The read noise distributions present a mean value of $262 \pm 1 e^{-}$and $887 \pm 1 e^{-}$with a standard deviation of $168.14 \pm 0.01 e^{-}$(64\% of the mean) and $451.75 \pm 0.07 e^{-}$( $50 \%$ of the mean) for Sub-Pixel and Pixel arrays respectively. The conversion gain distributions feature a mean value of $59 \pm 1 e^{-}$and $296 \pm 1 e^{-}$with a standard deviation of $20.94 \pm 0.02 e^{-}$ (35\% of the mean) and $90.14 \pm 0.08 e^{-}$(30\% of the mean) for Sub-Pixel and Pixel arrays respectively.

From the Central Limit theorem it can be shown that a random variable might be modeled as log-normal if it can be written as the multiplicative product of many independent random variables each of which is positive (Aitchison 1957). In this case, both conversion gain and read noise can be considered quantities which arise from a series of different amplification and conversion stages, i.e. multiplicative stages. In fact, as radiation interacts with the sensor, charge, generated inside the depletion region or reaching this region due to thermal diffusion, is collected. Collected charge is then multiplicatively amplified by a series of amplifiers both inside the pixel and on the sensor periphery, before being sampled and digitalized. This process is summarized in Equation (1) and schematically represented in Figure 4.

All the multiplicative factors of Equation (1) can be considered random variables assumed to be normally distributed for each pixel, and the total distribution across the entire matrix is still normal, due to the Central Limit theorem. Thus is possible to schematize the signal generation process as a multiplicative product of many normally distributed random variables. Hence, conversion gain and read noise, which are proportional to signal, can be modeled as global log-normal distributions.

Comparing the fit parameters in Table 3, it can be observed that the relative width $(\sigma / \mu)$ of the two distributions, namely conversion gain and read noise, are comparable for the two pixel types. In fact the relative width for conversion gain is $50 \%$ and $60 \%$, while these figures are $30 \%$ ad $35 \%$ for read noise for Pixels and Sub-Pixel respectively. This is expected since the width of these distributions is due to the same manufacturing variations for both pixel types .

\subsection{Photon Transfer Curve in stitching blocks}

The conversion gain and read noise matrices have been averaged in each of the $35 \mathrm{E}$ type stitching blocks constituting the sensitive regions of the detector (seeFigure 2). The percentages of variation per block with respect to the average value on the entire matrix are displayed in Figure 7 for both Pixel and Sub-Pixel arrays. The average percentage of variation is $1.31 \%$ for the conversion gain of Pixels and $1.18 \%$ for SubPixels, whereas it is $1.32 \%$ for the read noise of Pixels and $1.52 \%$ for Sub-Pixels, with 
a coefficient of variation $(\mathrm{COV}) \leq 1.9 \%$ for all of them. Per block variation in terms of read noise and conversion gain are comparable for both pixel types as expected.

This latest result is comparable with the analysis on non-uniformity reported by (Zin et al. 2010), who showed that gain and read noise of the LAS sensor, calculated per stitching block, exhibit a COV of the order of $3.79-7.02 \%$ and $3.85-5.67 \%$ respectively. When these COV values are compared with a value $\leq 1.9 \%$ measured for DynAMITe, this demonstrates a higher level of uniformity in terms of optical performance.

\subsection{Fixed Pattern Noise}

The level of FPN is shown in Figure 8 for Pixels $(a)$ and Sub-Pixels $(b)$ at half saturation. C-C and P-P FPN values have been calculated in different regions of the sensor: "whole array" refers to the FPN calculated on the full imaging area, "column output" refers to the FPN calculated in one of the seven column outputs of the sensor, "row sub-stitch" refers to the FPN calculated in a region of the column outputs limited in one of the five row stitching areas. The C-C FPN has been measured as $1.3 \%$ for Pixels and $2.0 \%$ for Sub-Pixels, whereas the P-P FPN results in $2.2 \%$ for Pixels and $4.2 \%$ for Sub-Pixels on the whole array.

FPN, calculated on the whole array, is globally higher for the Sub-Pixel than for the Pixel arrays. This is due to the total diode node capacitance of Sub-Pixels being dominated by the diode itself which suffers higher variations leading to more mismatches among pixels. Limiting the region for measurement to a column output or row sub-stitch leads to a decrease in FPN. C-C FPN is reduced by a factor 0.4 in the column output and by a factor of 0.6 in a row sub-stitch for the Sub-Pixels, whereas these values are 0.5 and 0.7 respectively for the Pixels. P-P FPN is reduced by a factor of 0.5 in the column output and by a factor of 0.6 in the row sub-stitch for the Sub-Pixels. However, Pixels do not show any significant decrease of P-P FPN confining calculation in limited regions (column output or row sub-stich), as P-P FPN for Pixels is dominated by the low-pass filter mentioned above.

Moreover, FPN, when evaluated for each of the 35 sensor stitching blocks (Figure 9), results in higher values at the edges of the sensor, e.g. stitching block 1 and 35 , attributed to higher manufacturing variation in the CMOS processes at the edge of the wafer. C-C FPN shows a similar trend of variation among stitching blocks for Sub-Pixels and Pixels (Figure 9 a). This can be explained taking into account the column parallel read-out and the fact that column amplifiers of both type of pixels are placed close to each other. Hence variations at amplifier level for one diode type could result in similar variations for the amplifiers of the other diode type, thus leading to a similar behavior in the columnar spatial variations, i.e. C-C FPN. Moreover both C-C and P-P FPNs show a periodic variability with a period of 5 stitching blocks, corresponding to the number of row stitching block per column. Thus a higher FPN is related to those stitching blocks which lay at the bottom of the sensor (blocks 5, 10, 15, 20, 25, 30, 35 in Figure 2) which may be representative a region of higher process variation being nearer the edge of the original wafer. 


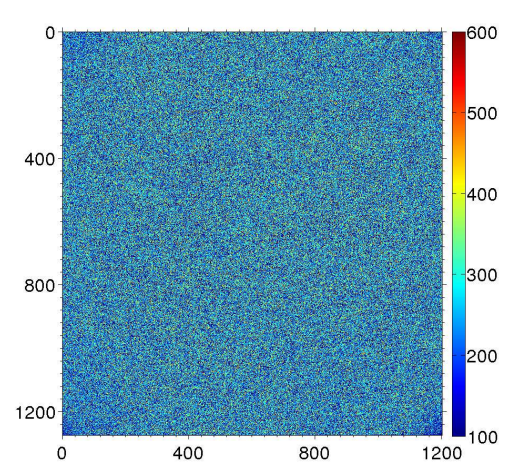

(a) Gain-P

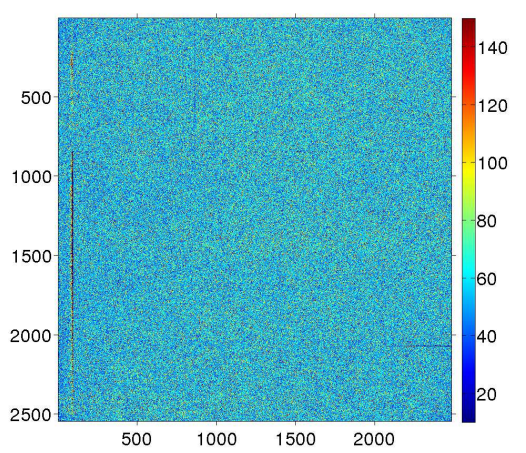

(c) Gain-SP

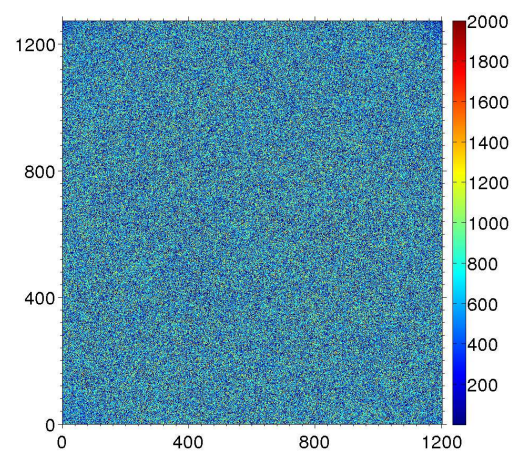

(e) Noise-P

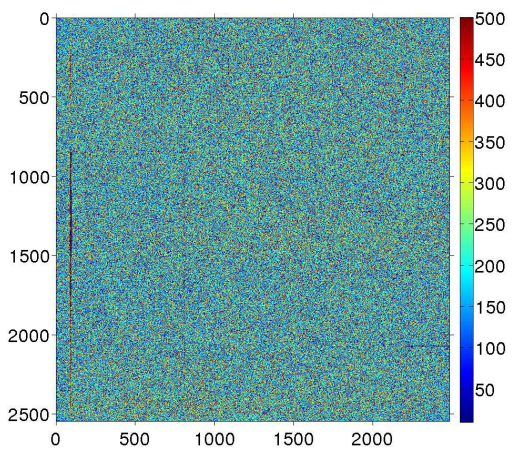

(g) Noise-SP

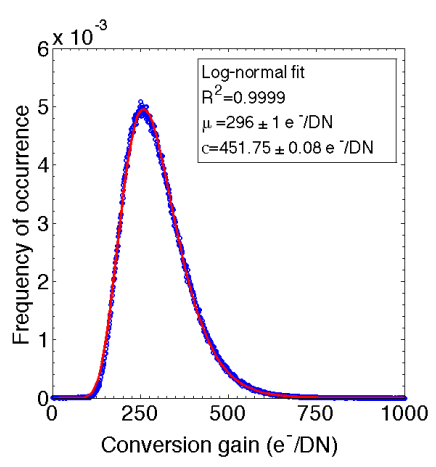

(b) Gain-P

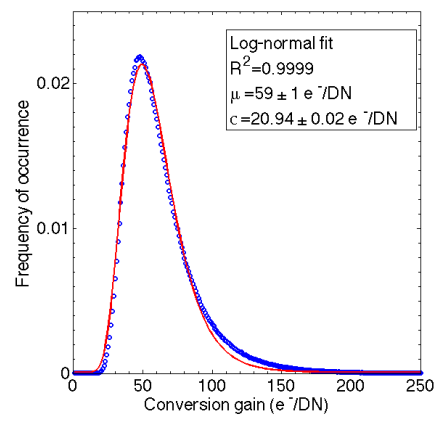

(d) Gain-SP

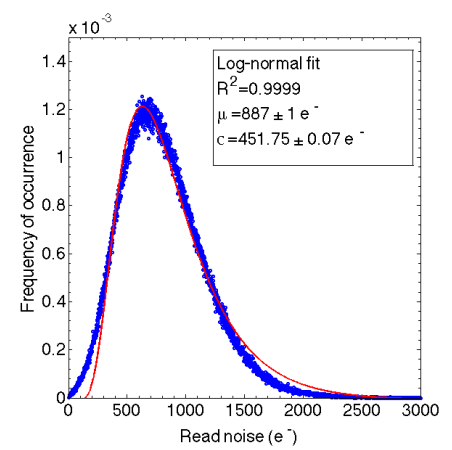

(f) Noise-P

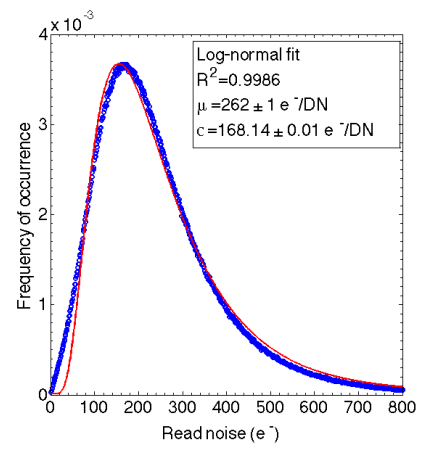

(h) Noise-SP

Figure 6. Read noise and conversion gain matrices for Pixels (inset a) and c)) and for the Sub-Pixels (inset $e$ ) and $f$ )) respectively, resulting from the per-pixel photon transfer curve. Color scales represent the conversion gain value per-pixel expressed in $e^{-} / D N$ (inset $a$ ) and $e$ )) and the read noise per-pixel in expressed $e^{-}$(inset $c$ ) and $\left.h\right)$ ). The relevant histogram for each of the matrices is reported (inset $b), d), g$ ) and $h$ )). 


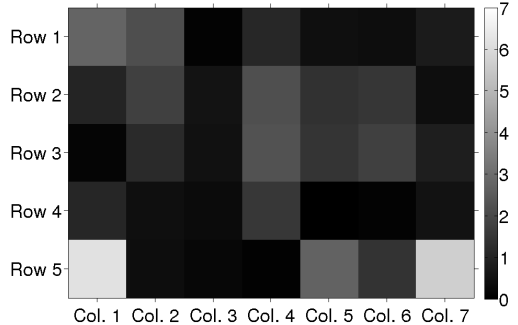

(a) Gain-P

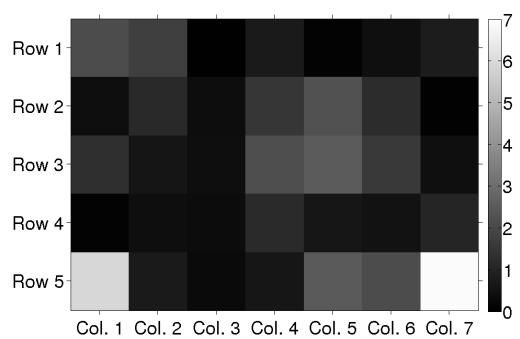

(c) Noise-P

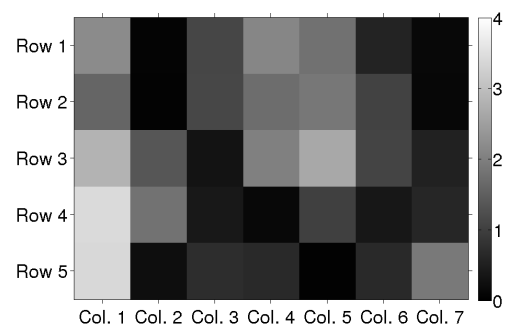

(b) Gain-SP

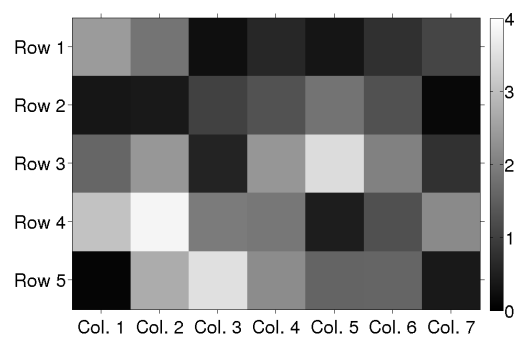

(d) Noise-SP

Figure 7. Percentage of variation of the read noise and conversion gain per stitching block with respect to the average value across the whole matrix for Pixels (inset $a$ ) and $d$ )) and for the Sub-Pixels (inset $b$ ) and $d$ )) respectively. Gray scales represent the percentage of variation with respect to the average value on the whole matrix expressed in $\%$.

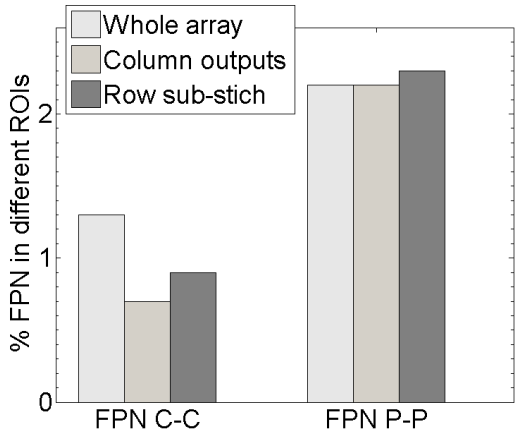

(a) FPN-P

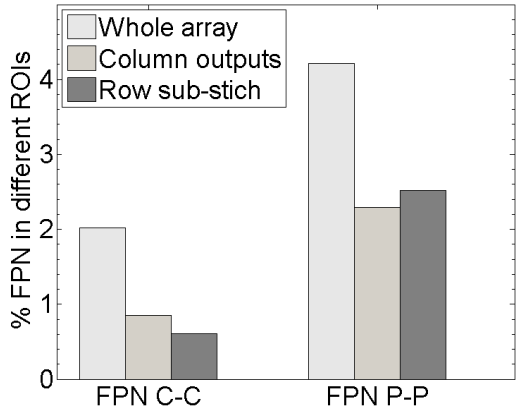

(b) FPN-SP

Figure 8. Percentage of FPN, both C-C and P-P, measured in different regions of the Dynamite detector for Pixels $(a)$ and Sub-Pixels $(b))$. 


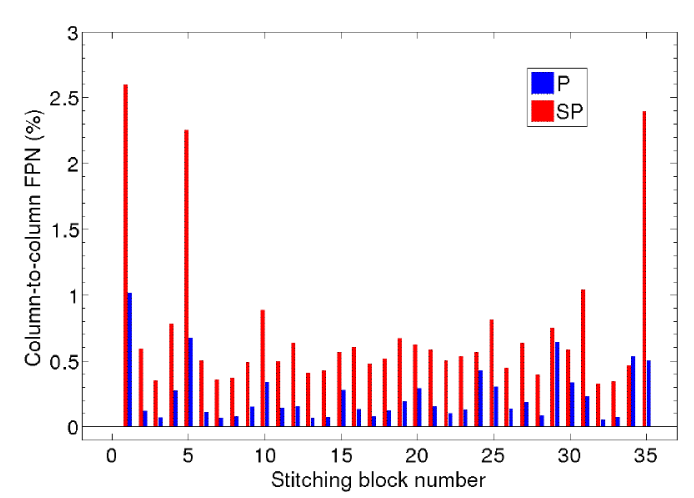

(a) C-C FPN

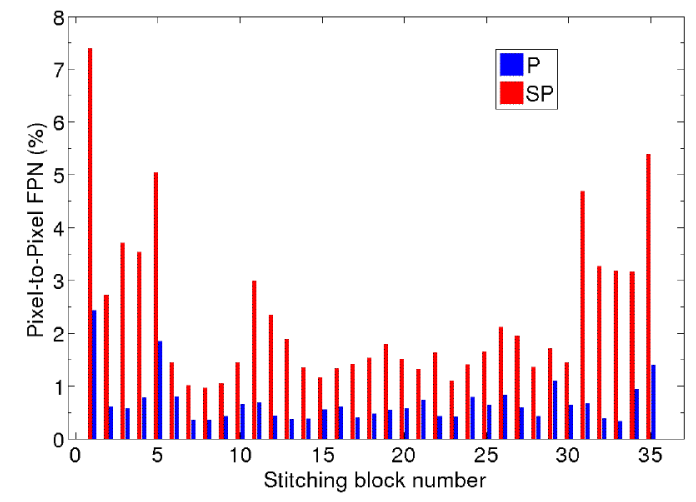

(b) P-P FPN

Figure 9. Percentage of Fixed Pattern Noise (FPN), both column-to-column (CC) $(a)$ and pixel-to-pixel (P-P) $(b)$, measured in each of the 35 stitching blocks of the Dynamite detector for Pixels and Sub-Pixels

\subsection{Contrast-to-Noise Ratio}

A further stage in this investigation led to test this system in a mammographic configuration, in order to assess to what degree detector non-uniformities affect image quality in a typical radiographic application. Figure 10 a) displays a composite image, obtained by averaging single frames, showing the Perspex test object being imaged in different positions to cover all the stitching blocks of this sensor. Object and background signals and standard deviations are then calculated to assess the CNR across the stitching blocks, after correcting for gain variations (Seibert et al. 1998). The top seven stitching blocks of the sensor showed some device-related sensitivity, and have been therefore discarded from analysis. CNR per stitching block is reported in Figure $10 b$ ) for Pixels and Sub-Pixels.

On average CNR results were derived to be higher for Pixels (average value of 34.4) compared to Sub-Pixels (average value of 5.9), with a COV of $13 \%$, compared to $26 \%$ COV for Sub-Pixels across all the 35 stitching blocks. This difference can be explained by the higher FPN Sub-Pixels feature, due to the total diode node capacitance being dominated by the diode itself, compared to Pixels. The uniformity of the CNR across both pixel arrays $\left(C N R_{u n i}\right)$ has been evaluated according to Equation (15), resulting in $79 \%$ uniformity for Pixels and $37 \%$ for Sub-Pixels.

The results obtained in terms of CNR and uniformity of CNR have been compared with the state-of-the-art large area sensors for mammography. A comparative analysis for CNR and uniformity is reported in (Baldelli et al. 2009) for a number of mammography system used in BreastCheck, the National Breast Screening Program of Ireland. The mammography systems investigated in the referenced work are: GE Senographe DS and GE Senographe Essential (Ghetti et al. 2008), both a-Si flat panels coupled to a CsI scintillator, Lorad Selenia(Young et al. 2007), an a-Se flat panel used for direct detection, and Sectra MDM-L30 and M-40 (Young et al. 2008), both based on the use of strip sensors in direct detection. Comparative data are shown in Table 4 together with some of the experimental settings. The thickness of the Perspex test 


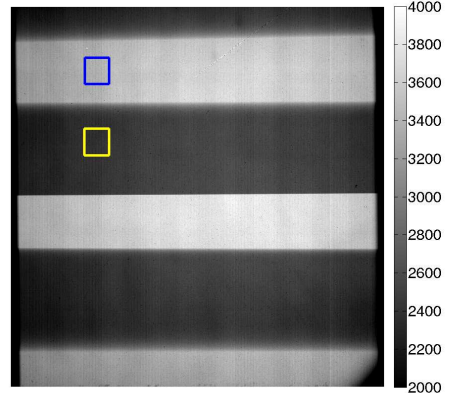

(a)

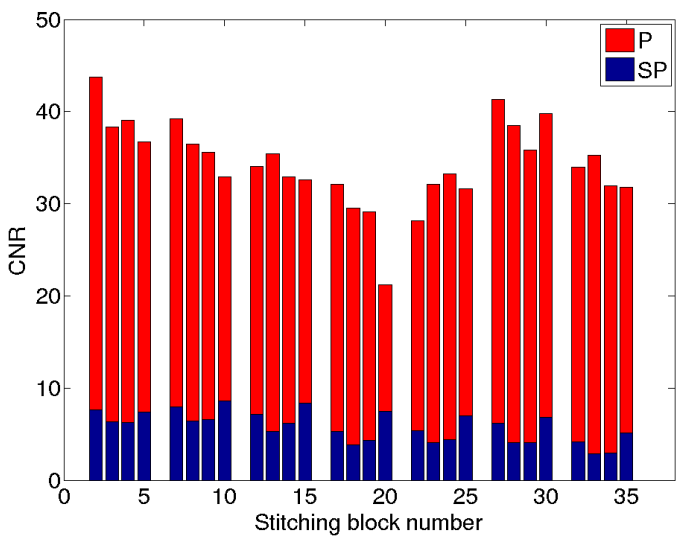

(b)

Figure 10. (a) Composite image displaying the Perspex test object used for CNR measurements stepped across the pixel array. Areas where signal and background are calculated in a single stitching block are displayed in blue and yellow respectively.c)CNR per stitching block for both Pixels (P) and Sub-Pixels (SP).

Table 4. Comparative data for CNR and uniformity in CNR reported for a number of large area detectors used in mammography and for the DynAMITe CMOS APS. All systems are tested with a $45 \mathrm{~mm}$ thick Perspex test object.

\begin{tabular}{llllll}
\hline Detector & Type $\mathrm{n}$ & $\mathrm{T} / \mathrm{F}$ & $\mathrm{kVp}$ & $C N R_{\text {mean }}$ & $C N R_{\text {uni }}(\%)$ \\
\hline GE DS & a-Si/CsI & $\mathrm{Rh} / \mathrm{Rh}$ & 29 & 8.14 & 96.2 \\
GE Essential & a-Si/CsI & $\mathrm{Rh} / \mathrm{Rh}$ & 29 & 12.83 & 88.9 \\
Lorad Selenia & a-Se/Direct & $\mathrm{Mo} / \mathrm{Mo}$ & 28 & 11.09 & 89.3 \\
Sectra MDM D40 & Si SS/Direct & W/Al & 29 & 2.47 & 36.1 \\
Sectra MDM L30 & Si SS/Direct & W/Al & 35 & 3.65 & 45.6 \\
DynAMITe & APS/CsI & Mo/Mo & 25 & 34.4 & 79 \\
Pixels & & & & & \\
DynAMITe & APS/CsI & Mo/Mo & 25 & 5.9 & 37 \\
Sub-Pixels & & & & & \\
\hline
\end{tabular}

object $(45 \mathrm{~mm})$ is not reported in the table, as it is the same for all the systems used in this comparison.

The Pixels of the DynAMITe sensor show the highest mean CNR when compared with the other detection systems of Table 4 (34.4). $C N R_{\text {mean }}$ is lower for Sub-Pixels (5.9) than that of FPIs (GE DS, GE Essential and Lorad Selenia), but results higher than that of the Sectra detectors. The CNR uniformity for Pixels is comparable with the highest among the $C N R_{u n i}$ values for the systems included in Table 4 (GE DS, GE Essential and Lorad Selenia). On the other side, the CNR uniformity for SubPixels is comparable with that achieved by the strip detectors (Sectra MDM-L30 and $\mathrm{M}-40)$. 
Table 5. Summary of imaging conditions and detector parameters used for the $\mathrm{DQE}(0)$ calculations.

\begin{tabular}{|c|c|c|c|c|}
\hline Application & $\begin{array}{l}\text { Energy } \\
(\mathrm{kVp})\end{array}$ & $\begin{array}{l}\text { Exposure } \\
(\mathrm{mR})\end{array}$ & Anode & Filtration \\
\hline Radiology & 110 & $0.03-3$ & $\mathrm{~W}$ & $2.75 \mathrm{~mm} \mathrm{Al}$ \\
\hline Detector & Technology & $\begin{array}{l}\text { Pixel pitch } \\
(\mu \mathrm{m})\end{array}$ & $\begin{array}{l}\sigma_{R} \\
\left(\mathrm{e}^{-}\right)\end{array}$ & Fill factor \\
\hline DynAMITe SP & APS & 50 & 150 & 0.7 \\
\hline DynAMITe P & APS & 100 & 780 & 0.7 \\
\hline (Jee et al. 2003) & a-Si FP & 97 & 2000 & 0.45 \\
\hline (Siewerdsen et al. 1997) & a-Si FP & 127 & 5000 & 0.8 \\
\hline (Jaffray and Siewerdsen 2001) & a-Si FP & 400 & 4000 & 0.8 \\
\hline
\end{tabular}

\subsection{Detective Quantum Efficiency}

Evaluation of detector performance is typically carried out using different methodology for APSs and FPIs. The former are usually studied by means of their photon transfer properties for evaluation of read noise, conversion gain and FPN (Janesick 2001, EMVA 2005), as in this work. The latter are investigated using DQE, Noise Power Spectrum and Modulation Transfer Function (Metz et al. 1995), in application-like conditions. For this reason a direct comparison between performance parameters measured for the APS used in this work and reported in literature for FPIs might not be directly achievable. To address this point a theoretical calculation of the DQE at zero-frequency, based on detector performance parameters such as read noise, fill factor and pixel pitch, has been undertaken comparatively for the DynAMITe detector and a number of FPIs.

Calculations of DQE(0) have been performed, following Equation (19), for the DynAMITe detector and for a number of FPIs, under radiology imaging conditions. Detectors involved in the comparison are a-Si flat panels reported in (Jee et al. 2003, Siewerdsen et al. 1997, Jaffray and Siewerdsen 2001). Parameters used for the DQE(0) calculation, such as pixel pitch, read noise and fill factor are reported in Table 5 together with the X-ray settings used. The same scintillator $(150 \mu \mathrm{m}$ thick CsI) was used for calculation of $\operatorname{DQE}(0)$ for all the detectors involved in the comparison. In fact, as $\operatorname{DQE}(0)$ (Equation (19)) depends on both scintillator-related $\left(\bar{g}_{1}, \bar{g}_{2}\right.$ and $\left.\epsilon_{g_{2}}\right)$ and detector-related parameters $\left(\sigma_{R}, \bar{g}_{4}\right.$ and $\left.a_{\text {pixel }}\right)$, the use of the same conversion system allows to exclude the interaction and conversion stages from the comparison, which effectively becomes a comparison of the effects of detector-related parameters.

The dependence of the $\mathrm{DQE}(0)$ with exposure for all the detectors involved in the comparison is shown in Figure 11. For low exposure values $\left(\bar{q}_{0}\right.$ in Equation (19)), the differences in read noise dominate the DQE and curves in Figure 11 show a significative difference between each other. At higher exposure values those differences tend to flat out as the number of X-ray quanta dominate the additive term in the denominator of Equation (19), and the scintillator efficiency and quantum gain represent detection limit of the system.

Pixels and Sub-Pixels of the DynAMITe detector show the highest detection efficiency among the detectors used in this comparison, due to the inherently lower noise of APS compared to FPIs. Sub-Pixels have the highest efficiency, because of 


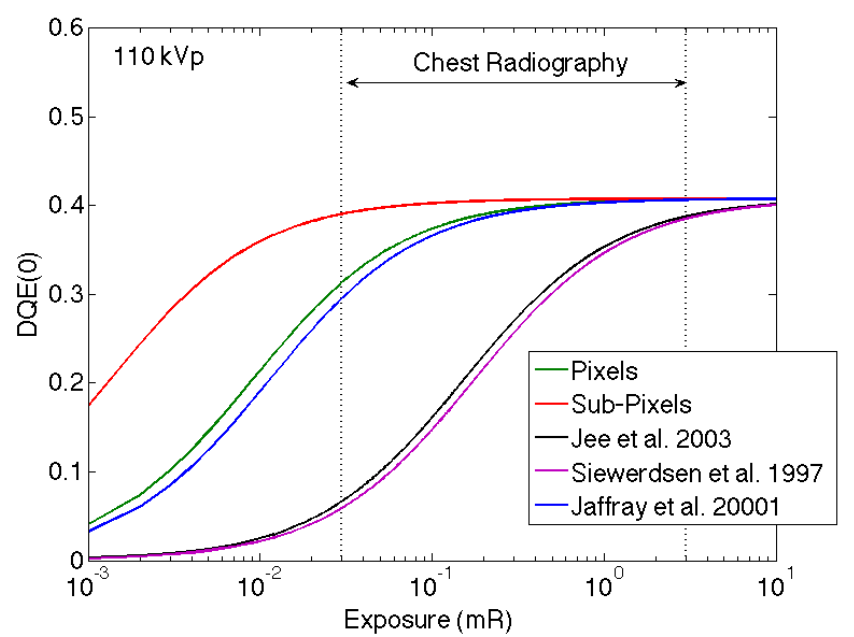

Figure 11. Calculated DQE $(0)$ versus exposure for the DynAMITe Pixels and Sub-Pixels and for FPIs under radiological imaging conditions. The typical exposure range of a specific application is highlighted in figure

their noise level which is the lowest among the detectors used in this comparison (see Table 5). $\mathrm{DQE}(0)$ for Pixels and for the flat panel reported in (Jaffray and Siewerdsen 2001) are comparable, even if subject to a very different noise level (100 and $4000 \mathrm{e}^{-}$respectively). This results from the effect of a larger pixel pitch $(400 \mu \mathrm{m})$ balancing off the effect of a higher noise in Equation (19). Flat panels presented by (Jee et al. 2003) and (Siewerdsen et al. 1997) have a comparable DQE, the lowest in the comparison, since it is dominated by a high noise (2000 and $5000 \mathrm{e}^{-}$respectively) on a relatively small pixel pitch (97 and $127 \mu \mathrm{m}$ respectively).

\section{Conclusion}

A new wafer scale CMOS APS, based on the use of different full well capacity diodes and capable of simultaneous low noise and high dynamic range, has been presented in this paper. An investigation into the uniformity of response of this sensor and regional variations has been carried on by means of electro-optical performance assessment and $\mathrm{X}$-ray imaging.

For the first time a per-pixel analysis of the electro-optical performance of a wafer scale CMOS APS has been carried out, to address inhomogeneity issues arising from the stitching techniques used to manufacture wafer scale sensors. A complete model spanning the signal generation in the pixel array, from charge generation inside the pixel sensitive volume to the digitization of the generated signal, has been provided and proved capable of accounting for noise and gain measured distributions.

Evaluation of uniformity in terms of noise and conversion gain highlighted a similar degree of homogeneity for both pixel types, being due to the same lithographic processes, while Fixed Pattern Noise showed an higher degree of variation, being ascribed to the electronic design of the pixels.

When tested in a conventional X-ray radiology set-up (i.e. mammography), the 
DynAMITe detector showed a uniformity in terms of CNR among the highest when compared with mammography detectors commonly used in clinical practise.

Finally a theoretical calculation of DQE at zero-frequency allowed comparison of the detection performance of the DynAMITe detector with FPIs, used in the medical imaging field. Evaluation of DQE showed how the intrinsic lower read noise of APS, compared to FPIs, results in higher DQE performance.

Considering the performance parameters assessed for this detector, in comparison with digital detectors commonly used in the clinical practise, demonstrates how such large area sensor technology may be successfully employed in medical imaging.

\section{Acknowledgments}

This work was supported by the EPSRC Multidimensional Integrated Intelligent Imaging Plus (MI-3 Plus) programme (EP/G037671/1).

\section{References}

Agranov G, Gilton T, Mauritzson R, Boettiger U, Altice P, Shah J, Lad J, Fan X, Brady F, McKee J, Hong C, Li X and Patrick J 2005 Optical-Electrical Characteristics of Small, Sub- $4 \mu \mathrm{m}$ and Sub-3 $\mu$ m Pixels for Modern CMOS Image Sensors in 'IEEE CCD and AIS Workshop Proc.' Vol. 3422 pp. 206-209.

Aitchison, J.and Brown J A 1957 The Lognormal Distribution Cambridge University Press.

Arvanitis C D, Bohndiek S E, Royle G, Blue A, Liang H, Clark A, Prydderch M and Speller R T R 2007 Empirical electro-optical and X-ray performance evaluation of CMOS active pixels sensor for low dose, high resolution X-ray medical imaging Med. Phys. 34(12), 4612-4625.

Ay S U and Fossum E R 2006 A $76 \times 77 \mathrm{~mm}^{2}$, 16.85 Million Pixel CMOS APS Image Sensor in 'VLSI Circuits, Digest of Technical Papers. 2006 Symposium on' pp. 19-20.

Baldelli P, Phelan N and Egan G 2009 A novel method for contrast-to-noise ratio (CNR) evaluation of digital mammography detectors Eur. Radiol. 19, 2275-2285.

Beecken B P and Fossum E R 1996 Determination of the conversion gain and the accuracy of its measurement for detector elements and arrays Appl. Opt. 35(19), 3471-3477.

Bigas M, Cabruja E, Forest J and Salvi J 2006 Review of CMOS image sensors Micr. Journ. 37(5), 433 $-451$.

Bohndiek S, Blue A, Cabello J, Clark A, Guerrini N, Evans P, Harris E, Konstantinidis A, Maneuski D, Osmond J, O'Shea V, Speller R, Turchetta R, Wells K, Zin H and Allinson N 2009 Characterization and Testing of LAS: A Prototype 'Large Area Sensor' With Performance Characteristics Suitable for Medical Imaging Applications IEEE Trans. Nucl. Sc. 56(5), 29382946.

Colbeth R 2010 Solar Cells and their Application Hoboken, NJ, USA.

Cunningham I A, Westmore M S and Fenster A 1994 A spatialfrequency dependent quantum accounting diagram and detective quantum efficiency model of signal and noise propagation in cascaded imaging systems Med. Phys. 21, 417-427.

Dance D R, Strudley C J, Young K C, Oduko J M, Whelehan P J and Mungutroy E H L 2012 in A Maidment, P Bakic and S Gavenonis, eds, 'Breast Imaging' Vol. 7361 of Lecture Notes in Computer Science Springer Berlin Heidelberg pp. 316-321.

El Gamal A and Eltoukhy H 2005 CMOS image sensors Circuits and Devices Magazine, IEEE $\mathbf{2 1}(3), 6-20$.

El Gamal A, Fowler B, Min H and Liu X 1998 Modeling and Estimation of FPN Components in CMOS Image Sensors Proc. SPIE 3301, 168-177.

EMVA 2005 'European Machine Vision Association Standard 1288 Standard for Characterization and Presentation of Specification Data for Image Sensors and Cameras'.

Esposito M, Anaxagoras T, Fant A, Wells K, Konstantinidis A, Osmond J P F, Evans P M, Speller R D and Allinson N M 2011 DynAMITe: a wafer scale sensor for biomedical applications Journ. Inst. 6(12), C12064.

Esposito M, Anaxagoras T, Wells O D K and Allinson N M 2012 Radiation hardness of a large area CMOS active pixel sensor for bio-medical applications in 'Nuclear Science Symposium and Medical Imaging Conference (NSS/MIC), 2012 IEEE' pp. 1300-1304. 
Farrier M, Achterkirchen T G, Weckler G and Mrozack A 2009 Very Large Area CMOS Active-Pixel Sensor for Digital Radiography Elec. Dev., IEEE Transactions on 56(11), 2623-2631.

Fish A and Yadid-Pecht O 2004 CMOS Imagers: From Phototransduction to Image Processing Kluwer Academic Publishers.

Fossum E R 1995 CMOS image sensors: electronic camera on a chip in 'Electron Devices Meeting, 1995. IEDM '95., International' pp. 17-25.

Ghetti C, Borrini A, Ortenzia O, Rossi R and Ordatez P L 2008 Physical characteristics of GE Senographe Essential and DS digital mammography detectors Medical Physics 35(2), 456463.

Hamamatsu 2013 'X-Ray Flat Panel Imagers for Radiology' http://www.hamamatsu.com/us/en/product/category/3100/4011/4157/index.html. Accessed October 23, 2013.

Holst G and Lomheim T 2011 CMOS/CCD Sensors and Camera systems SPIE Press.

Jaffray D and Siewerdsen J 2001 Volumetric Cone-Beam CT System Based on a $41 \times 41 \mathrm{~cm}^{2}$ FlatPanel Imager in 'Proc. SPIE' Vol. 4320 pp. 800-807.

Janesick J R 2001 Scientific Coupled Devices SPIE Press.

Jee K, Antonuk L, El-Mohri Y and Zhao Q 2003 System performance of a prototype flat-panel imager operated under mammographic conditions Med. Phys. 26(7), 1874-1890.

Konstantinidis A C, Olivo A, Munro P R, Bohndiek S E and Speller R D 2010 Optical characterisation of a CMOS active pixel sensor using periodic noise reduction techniques Nucl. Instrum. Methods A 620(23), $549-556$.

Konstantinidis A C, Szafraniec M B, Speller R D and Olivo A 2012 The Dexela 2923 CMOS Xray detector: A flat panel detector based on CMOS active pixel sensors for medical imaging applications Nucl. Instrum. Methods A 689(0), $12-21$.

Korthout L, Verbugt D, Timpert J, Mierop A, de Haan W, Maes W, de Meulmeester J, Muhammad W, Dillen B, Stoldt H, Peters I and Fox E 2009 A wafer-scale CMOS APS imager for medical X-ray applications in 'International Image Sensor Workshop, Bergen, Norway' number 70.

Lacoe R 2008 Improving Integrated Circuit Performance Through the Application of Hardness-byDesign Methodology Nucl. Sc., IEEE Trans. on 55(4), 1903-1925.

Lo Y 1998 Solid state image sensor: technologies and applications in 'Proc. SPIE' Vol. 3422 pp. 70-80.

Metz C, Wagner R, Doi K, Brown D, Nishikawa R and Myers K 1995 Toward consensus on quantitative assessment of medical imaging systems Med. Phys. 22, 1057-1061.

Osmond J, Zin H, Harris E, Lupica G, Allinson N and Evans P 2008 Imaging of moving fiducial markers during radiotherapy using a fast, efficient active pixel sensor based EPID Med. Phys. 38(11), 6152-6159.

RadIcon 2013 'RadEye1' http://www.rad-icon.com/products-radeye.php/. Accessed October 23, 2013.

Reshef R, Leitner T, Alfassi S, Sarig E, Golan N, Berman O, Fenigstein A, Wolf H, Hevel G, Vilan $\mathrm{S}$ and Lahav A 2009 Large-Format Medical X-ray CMOS Image Sensor for High Resolution High Frame Rate Applications in 'Proceedings of International Image Sensor Workshop'.

Rowlands J and Yorkston J 2000 Handbook of Medical Imaging Vol. 1 of Physics and Psychophysics SPIE Bellingham, WA.

Scheffer D 2007 A Wafer scale active pixel CMOS image sensor for generic x-ray radiology in 'Proc. SPIE' Vol. 6510 pp. 65100O-65100O-9.

Seibert J A, Boone J M and Lindfors K K 1998 Flat-field correction technique for digital detectors in 'Proc. SPIE' Vol. 3336 pp. 348-354.

Siewerdsen J, Antonuk L, Mohri Y E, Yorkston J, Huang W, Boudry J M and Cunningham I A 1997 Empirical and theoretical investigation of the noise performance of indirect detection, active matrix flat-panel imagers (AMFPIs) for diagnostic radiology Med. Phys. 24, 71-89.

Siewerdsen J H and Jaffray D A 1999 A ghost story: Spatio-temporal response characteristics of an indirect-detection flat-panel imager Med. Phys. 26(8), 1624-1641.

Stark B, Noelting B, Jahn H and Andert K 1992 Method for determining the electron number in charge-coupled measurement devices Opt. Eng. 31(4), 852-856.

Swank R K 1973 Absorption and noise in X-ray phosphors J. Appl. Phys. 44, 4199-4203.

Turchetta R, Guerrini N and Sedgwick I 2011 Large area CMOS image sensors Journ. of Inst. 6(01), C01099.

Weisfield R L and Bennett N R 2001 Electronic noise analysis of a 127-m pixel TFT photodiode array Proc. SPIE 4320, 209-218.

Young K C, Oduko J M and Alsager A 2008 Technical Evaluation of the Sectra MDM-L30 Full Field Digital Mammography System in 'NHS Cancer Screening Programmes, NHSBSP Equipment Report 0805'. 
Young K, Oduko J M and Woolley L 2007 Technical Evaluation of the Hologic Selenia Full Field Digital Mammography System in 'NHS Cancer Screening Programmes, NHSBSP Equipment Report 0701'.

Zentai G and Colbeth R 2012 Pros and cons of CMOS X-ray imagers in 'Medical Measurements and Applications Proceedings (MeMeA), 2012 IEEE International Symposium on' pp. 1-5.

Zhao W, Ristic G and Rowlands J 2004 X-ray imaging performance of structured cesium iodide scintillators Med. Phys. 31(9), 2594-2605.

Zin H M, Konstantinidis A C, Harris E J, Osmond J P, Olivo A, Bohndiek S E, Clark A T, Turchetta R, Guerrini N, Crooks J, Allinson N M, Speller R and Evans P M 2010 Characterisation of regional variations in a stitched CMOS active pixel sensor Nucl. Instrum. Methods A 620(23), $540-548$. 